\title{
PRESENCIA VALENCIANA EN EL SITIO DE BALAGUER. AÑO 1413
}

Desamparados Pérez Pérez

En la sección de Cancillería Real del Archivo del Reino de Valencia (ARV) hay un volumen en cuyo primer folio se lee Registre dels actes seguits per la subvencio feta per los dels tres staments del regne de Valencia dels CCC homens a cavall proferts e trameses al Senyor Rey y, añadido posteriormente, $e$ dels cinchcents derrerament proferts, el cual va a servir de base a este trabajo'.

Más que un volumen, son 48 folios numerados actualmente, cosidos a un lomo de pergamino, de los cuales hay nueve en blanco. Ha aparecido entre documentación varia que se estaba ordenando, el cual por su contenido debía incorporarse a la sección de Generalidad, ya que recoge las actas de un parlamento celebrado por la Diputación del General en 1413, para ayudar a Fernando I en el asedio a Balaguer, donde se había refugiado el conde de Urgel. Sin embargo, como todos los procesos de Cortes están incluidos desde antiguo en la Real Cancillería, se ha unido a ellos.

Su tamaño es de $300 \times 230 \mathrm{~mm}$ y la caja de escritura ocupa $240 \times 160 \mathrm{~mm}$. El papel, muy bueno, es todo de la misma cali-

I ARV, Real, libro 619 bis. 
dad, con los corondeles separados por una distancia de 35 a $40 \mathrm{~mm}$ y los puntizones, muy regulares, sobre 15 hilos por $20 \mathrm{~mm}$. Todo el papel lleva la misma filigrana, muy frecuente desde el primer cuarto del siglo xIv, y que se interpreta de muy distinta manera según los autores ${ }^{2}$. Está formada por tres elevaciones o cumbres sobre una línea horizontal, la cumbre central más elevada y separada de las laterales por dos depresiones iguales que no llegan a la base y rematada por una cruz, cuyo brazo más largo coincide con un corondel y el más corto con un puntizón, pero ambos son perfectamente visibles. Dada la frecuencia con que se prodiga esta filigrana no es posible señalar la procedencia del papel, porque las fábricas valencianas, catalanas e italianas la utilizan y en esta época es frecuente encontrar pagos por compra de papel toscano, por lo que puede tener cualquier origen.

La tinta utilizada es muy ferruginosa, pero dada la buena calidad del papel, éste no se ha afectado por el exceso de óxido, y se conserva perfectamente. La letra es minúscula bastarda, parte de ella muy cursiva y más caligráfica cuando se copian cartas literalmente. La lengua empleada es la valenciana, aunque hay parte de algún documento en latín.

El notario que escribe las actas, Jaime de Vallseguer, en ocasiones debe redactar de memoria o por lo que le han dicho otras personas, si es que él no ha estado presente en la sesión, porque hay expresiones tales como segons relacio a mi dit Jacme Vallseguer, notari, feta per Bernat de Bonshoms; o semblants paraules; o equivalents paraules ${ }^{3}$.

Las sesiones de este parlamento se celebran en la ciudad de Valencia, en la casa de la cofradía de San Jaime principalmente, pero también hay alguna sesión en la de la cofradía de San Narciso y en el convento de Predicadores.

Se inicia el manuscrito con la reunión del parlamento el día 21 de junio de 1413, previa autorización del portantveus de Go-

${ }^{2}$ Bкіquet, C.M. Les filigranes. Dictionnaire historique des marques du papier. $2^{*}$ ed. Nueva York 1966, vol. III, pp. 588-589.

${ }^{3}$ ARV, Real, libro 619 bis, fols. $10,11 \mathrm{v}^{\circ}, 15 \mathrm{v}^{\circ}$, etc. 
vernador Juan Escriva, dada el día 15 anterior, y la relación de todos los asistentes de los tres brazos o estamentos.

El objeto de la convocatoria es informar a los asistentes de las circunstancias en que se encuentran algunos territorios de la Corona, principalmente de Aragón y Cataluña, en donde rebeldes al rey ayudados por milicias extranjeras pretenden ocupar ciertos castillos y lugares de Aragón.

Unos días antes de la convocatoria, los diputados del General, al tener noticia de estos acontecimientos y porque desean una información más exacta, escriben a los jurados de Zaragoza, paers de Lérida y consellers de Barcelona ${ }^{4}$, solicitando información puntual de lo que ocurre y de las medidas que han tomado, porque ellos no se pueden quedar al margen de este hecho y necesitan tomar medidas y proveer lo que sea más conveniente con conocimiento de causa.

Los hechos no son otros que la rebelión del Conde de Urgel, porque con la solución del Compromiso de Caspe se vió eliminado de suceder a Martín el Humano e instigado por su madre, Margarita de Montferrato, y Antón de Luna en mayo de 1413 abiertamente declara su hostilidad a Fernando I, encerrándose, después de diversos reveses, en la ciudad de Balaguer, a la que el monarca aragonés pone sitio.

En la primera sesión de este parlamento se da cuenta de que el rey apurado por estas circunstancias fa congregacio de gents d'armes para enviar al reino de Aragón, ante la amenaza de invasión de tropas extranjeras que ayudan a Jaime de Urgel. Los diputados valencianos deseosos de prestar ayuda voluntariamente redactan unos capitulos en los que señalan la ayuda que el reino de Valencia ofrece al monarca, capítulos que este habrá de jurar, aprobar y confirmar para ponerlos en práctica, pero con la puntualización de que ni de las actuaciones a las que se obliguen en los capítulos ni de toda la ayuda que presten al rey se podrán inferir lesiones a las libertades, fueros, costumbres y de-

4 ARV, Generalidad, libro 4930 bis, fol. 705 r y v. Vid. Apéndice documental, núm. 1, en el que se incluye como ejemplo la carta que los diputados escriben a los jurados de Zaragoza. 
rechos del Reino, ni de los tres estamentos, ni de los particulares.

En estos capítulos se comprende la contratación de trescientos hombres armados y a caballo, por un período de tres meses y salario de 7 sueldos diarios. Habrán de ser naturales o vecinos del Reino, o estar al servicio de valencianos o vecinos de Valencia, y si no cumplen cualquiera de estos requisitos cobrarán cuando se haya completado el número de trescientos. Antes de cobrar deberán presentar su caballería y armas y posteriormente siempre que se les exija su capitán.

No podrán abandonar el servicio sin licencia de su capitán, si lo hicieren perderán armas, caballería y sueldo de un mes, e incluso deberán devolver lo cobrado si no hubieren prestado servicio. Además, no harán servicio distinto del previsto en estos capítulos, ni siquiera otras empresas que proponga el rey o las órdenes militares, como defensa de fronteras contra moros.

El salario de los soldados y gastos de la empresa se sacará de las generalitats, que era una de las formas previstas en la Diputación del General para sacar ingresos ${ }^{5}$, pero para disponer inmediatamente de dinero contante, se cargarán censales sobre ellas en cantidad suficiente para reunir 18.000 florines de oro de Aragón, dinero que se estima bastará para la empresa ${ }^{6}$.

También se prevé en estos capítulos, para no multiplicar el número de funcionarios y aumentar más los gastos, que los mismos clavarios y diputados que administran las generalitats administren este dinero y den cuenta a los jueces designados por las Cortes, como es costumbre hacerlo.

Ni el rey, ni su primogénito, ni cualquier funcionario, por amplia que sea su jurisdicción, podrá entrometerse en lo que concierne a la subvención, aunque surja algún pleito o recurso por causa de la misma, pero si los clavarios, diputados o administradores del General solicitan consejo o ayuda, se les de, a fin de cumplir felizmente cuanto se comprende en los capítulos.

5 Camarena, José: Función económica del «General de Regne de Valencian, en el siglo XV. Anuario de Historia del Derecho Español, tomo XXV, núm. 84 , p. 7.

6 Camarena, José: op. cit., p. 11. 
Se propone el nombramiento de capitán de este ejército a D. Alfonso, duque de Gandía, al que se le ha de reconocer toda la jurisdicción que corresponde en estos casos. Es notable que habiendo sido uno de los pretendientes a la Corona y por tanto, en cierta manera, rival de Fernando I, ahora los diputados le propongan capitanear este ejército, lo que él y el rey aceptarán sin recelo alguno.

El rey y su primogénito han de jurar y prometer cumplir estos capítulos en todos sus extremos para que la oferta se cumpla.

Todos los documentos que se hayan de extender por causa del cumplimiento de los capitulos quedarán exentos del derecho de sello.

Por su parte, los diputados firmarán cartas de inmunidad por las acciones en las que puedan verse involucrados a consecuencia del cargamento de censales, ya que éstos, su redención y el pago de los intereses de los censales, ha de hacerse sobre las generalitats?

Hay una voluntad manifiesta de ayudar al monarca de manera desinteresada, pero queda bien claro en los capítulos, que la oferta será realidad sólo en las condiciones que establece la Diputación del General y el rey y su heredero habrán de aceptar, jurar y confirmar.

Los diputados, por otra parte, preven que el rey se avendrá a firmar los capítulos porque en la misma sesión que se dan a conocer y se aceptan por todos los asistentes de los tres estamentos, sin esperar a la confirmación por parte del rey, se redacta el sindicado y la carta de gracia mediante los cuales los diputados podrán cargar censales sobre las generalitats para costear la empresa.

Igualmente en esta sesión se escribe una carta al duque de Gandía, D. Alfonso, comunicándole el acuerdo del Parlamento de nombrarle capitán del ejército que se ofrece al rey para ir en su ayuda al sitio de Balaguer, rogándole, además, que venga a Valencia lo antes posible para hacerse cargo de la empresa.

ARV, Real, libro 619 bis, fol. $3 \mathrm{v}^{\circ}$ y ss. Vid. Apéndice documental, núm. 2. 
En esta misma fecha, 21 de junio, se envian los capítulos al rey, acompañados de una carta con Pedro Desplugues, ya que los diputados no pueden disponer de cargar censales sobre las generalitats si el rey no les autoriza, autorización que se concreta en la aprobación de los capítulos; por ello, cuando el 4 de julio Fernando I escribe al duque de Gandía rogándole la mayor rapidez en el envío del ejército, quejándose de la dilación que pone, los diputados le contestan en el siguiente sentido: placia a vostra molt gran senyoria cuytar la ferma dels dits capitols per la manera contenguda e concordada per aquells, car sens aquella res no's pot desempaxar".

En la sesión del 5 de julio los diputados proponen a Pedro Gil, regente de la clavería del General, que anticipe 6.000 florines para pagar el primer mes a los soldados que se ofertan al rey, pero el clavario se muestra reticente y duda de que le sea posible acceder a ello, alegando que no constava tro açi quel molt alt senyor Rey hagues fermats, actorizats e aprobats los capitols concordats per los dits tres staments, pero los quals era donat poder de fer carregament de censals sobre lo dit general" y salva su responsabilidad accediendo al anticipo cuando se le promete por los asistentes a la reunión que entre todos pagarán cualquier daño que por ello le pueda sobrevenir, incluso el Baile ganeral, Juan Mercader, se obliga con las rentas reales para este mismo anticipo.

El 6 de julio, ante una comisión de cuatro diputados, se presenta Francisco Desplugues para entregar las cartas que su hermano Pedro ha traído urgentemente, cartas que ya eran esperadas, pues son la confirmación de los capítulos y una misiva indicando que se envían aquellos aprobados, rogando se le mande con presteza la tropa ofrecida. La confirmación es de fecha 30 de junio y la carta de envío de 2 de julio ${ }^{10}$.

8 ARV, Real, libro 619 bis, fol. $10 v^{\circ}$.

9 ARV, Real, libro 619 bis, fol. 11.

10 ARV, Generalidad, libro 681, fol. 87 y Generalidad, libro 2642, fol. 132, se registran el pago y ápoca del viaje desde Balaguer a Valencia trayendo la confirmación de los capítulos. 
Una vez aprobados por el rey los capítulos, no hay ninguna razón para que no salga el ejército hacia Balaguer y en este sentido los diputados escriben al Duque de Gandía el 18 de julio presionándole para que no retrase la salida, el cual promete hacer la presentación o mostra del ejército el próximo día 25 , fiesta de Santiago, y salir el 27 si ya ha cobrado cada soldado su salario"; sin embargo, vuelven a fallar los planes, porque la caballeria no está totalmente equipada y la mostra no se hará hasta el domingo siguiente, 30 de julio.

La presentación de este ejército debió ser un espectáculo por los detalles que se dan de ella: tuvo lugar en la explanada que había ante las puertas del Real delante de la Diputación del General y gran muchedumbre de gente que acudió a verla ${ }^{12}$. En esta presentación se reúnen 271 soldados, que relaciona nominalmente el acta del Parlamento, señalando los acompañantes que cada caballero lleva consigo, recibiendo de todos ellos el juramento de acatamiento y homenaje acostumbrados.

Todavía retrasará el duque su salida hasta el jueves siguiente, día 3, no obstante las presiones de los diputados que quieren ver cumplida su promesa al rey lo antes posible para evitarse responsabilidades y así se lo hacen saber al duque, obligando al notario Vallseguer que levante acta de esta presión, pero D. Alfonso alega que no ha podido salir antes porque su escribano se retrasó en pedir las acémilas que necesitaban.

En días posteriores, otros nobles con sus gentes partirán, después de haber jurado ante los diputados su acatamiento al duque de Gandia, para unirse al ejército. También ellos y sus acompañantes vienen relacionados en las actas.

Aunque los diputados con el duque de Gandía envian una carta

"ARV, Real, libro 619 bis, fol. 15. Hay un error de fecha en el día que dice del duque de Gandia que saldrá de Valencia, porque si la mostra la hace el dia de San Jaime, 25 de julio, martes, para salir el jueves siguiente, este dia debe ser 27 de julio y no 28 , como dice el documento.

12 ARV, Real, libro 619 bis, fol. $16 \mathrm{v}^{\circ}$. Vid. Apéndice documental, núm. 3. 
al rey con la oferta del ejército y otros asuntos relativos al reino, para que el monarca tenga noticia más rápida de que el ejército ya va en su ayuda, mediante correo urgente - ab correu cuytatescriben a Aznar Pardo de la Casta que está con el rey, para que le informe.

En el manuscrito que vamos siguiendo queda interrumpida toda información sobre la llegada y posteriores actuaciones de este ejército, pero los diputados, sin embargo, solicitan informes sobre lo que ocurre en el sitio de Balaguer, según referencias encontradas en otros documentos ${ }^{13}$.

Se reanudan las actas el 23 de octubre, porque nuevamente se reúne el Parlamento, ya que la empresa de Balaguer no ha terminado y la oferta de los 300 hombres durante tres meses, hecha al rey, llega a su fin. A ellos, además, se han unido otros nobles valencianos llegados posteriormente y si no se prevé a su manutención, se habrán de volver.

Ante los representantes de los tres estamentos, el notario Jacme de Vallseguer lee unos nuevos capítulos propuestos al rey para continuar la ayuda del Reino, salvando, como la primera vez, las libertades, fueros y franquicias del mismo.

La ayuda ahora se amplía hasta 500 hombres, que son los que se supone debe haber en el sitio de Balaguer, pero las pautas sobre las que se redactan los nuevos capítulos, apenas difieren de los que se aprobaron el 21 de junio: se abonará el salario de un mes a 500 hombres armados y a caballo, valencianos o vecinos de Valencia, salario que se podrá prorrogar otro mes, y el dinero para pagarles se sacará de las generalitats, mediante el establecimiento de censales en cantidad suficiente para conseguir 20.000 florines.

Todos los presentes aceptan y firma estos capítulos, el sindicado y las cartas de gracia para cargar los censales, enviando una misiva al rey rogándole jure y confirme los nuevos capí-

13 ARV, Generalidad, libro 681, fol. $89 v^{\circ}$ y Generalidad, libro 2642, fol. $143 v^{\circ}$ se registran el pago y ápoca de un viaje de Francesch de Alcala a Balaguer llevando una carta de los diputados a Amar Pardo solicitando noticias. 
tulos $^{14}$. Para evitar en lo posible los retrasos, también escriben a Juan de Aguilar, para que se tome el máximo interés y el monarca no dilate la confirmación de los capítulos.

El monarca, como era de esperar, agradece vivamente la prórroga de la ayuda: regraciants vos tant com podem la damunt dita graciosa e liberal subvencio, mediante una carta fechada en el sitio de Balaguer, el 29 de octubre, y en ella les recomienda que el baile general Juan Mercader lleva un encargo suyo, que espera acepten y le den crédito. Este encargo no es otro que la urgencia que tiene el rey de que se le envie el dinero para pagar el primer mes de la ampliación a los 500 hombres que desde Valencia colaboran en el asedio de Balaguer.

Para que no haya más dilaciones, se acuerda en el Parlamento enviar al clavario Pedro Gil, con 10.000 florines, acompañado de la escolta necesaria que asegure la llegada del dinero sin ningún riesgo. Además lleva un memorial al rey sobre ciertos asuntos relacionados con la subvención, al que el rey debe dar crédito, pues el monarca exige más allá de lo que los capitulos autorizan a los diputados y ellos se escudan en ellos para no ceder.

Mientras Pedro Gil marcha a Balaguer, se rinde la ciudad y cae preso el conde de Urgel. Los diputados felicitan al monarca por esta victoria y le comunican que ya ha salido Pedro Gil para pagar a los ejércitos valencianos. Pero ya por San Mateo, Pedro Gil empieza a encontrar gente de la que estuvo en el asedio de Balaguer que vuelve a Valencia, asaltándole la duda de si les debe pagar, porque en los capitulos se señalaba claramente que se pagara a todos los que estuvieran en Balaguer el día en que se abonara el sueldo a los soldados, consultando sobre ello a los diputados, que le responden que los que han vuelto porque el rey les autorizó, con la confianza de que se les pagaría, se les abone el mes de prórroga, pero los que voluntariamente han abandonado el lugar de Balaguer, no pueden cobrar.

Llegado Pedro Gil a Balaguer, tendrá serias dificultades para liquidar a las tropas, pues el rey ha anticipado dinero a los que

14 ARV, Real, libro 619 bis, fol. 24 y ss. 
autorizó a volver a Valencia, y Bernat de Centelles, que llevaba 123 hombres y Pedro Maça otros 100 le exigen el sueldo de todos, porque también han anticipado parte.

Nuevas cartas de los diputados el 13 de noviembre y 5 de diciembre orientarán a Pedro Gil sobre cómo debe actuar, ya que incluso algunos de los soldados pretenden cobrar en Valencia, pidiendo su salario a los diputados y se ha de proceder cuidadosamente para no duplicar gastos.

Cumplido su cometido, vuelve Pedro Gil a Valencia y presenta el día 12 al Parlamento el memorial de todos los gastos y pagos realizados, además de contar todos los trabajos que tuvo que soportar y las presiones del rey que pretendía se le entregara también el dinero del segundo mes, aunque ya Balaguer se había tomado y por los capítulos no correspondía ampliar la subvención un mes más.

El memorial tiene dos partes: la primera recoge las cantidades entregadas para pagar los sueldos de la tropa y la segunda todos los gastos del viaje a Balaguer para llevar el dinero; en una y otra señala con todo detalle a quién paga y cuánto paga, con indicación del tiempo contabilizado, añadiendo en cada partida que tiene ápoca que justifica el pago.

Entrega el dinero del ejército a los capitanes y así vemos que además del duque de Gandia con su tropa participaron en el sitio de Balaguer, Bernat de Centelles, acompañado de 93 hombres armados a caballo, Pedro Maça de Liçana con 73, Aznar Pardo con 5 y los hermanos Villarrasa con 12 . No hay relación nominal de cada uno de los que acompañan a estos nobles valencianos, como se relacionaron los que salieron al mando del duque de Gandia, según ya se indicó.

Sabemos que Bernat de Centelles y Pedro Maça de Liçana salieron de Valencia antes de que se convocara el Parlamento y el General del reino hiciera su oferta al monarca, porque el rey escribió al baile general, Juan Mercader, el 15 de mayo de 1413, para que le enviara un ejército, autorizándole a cargar censales que le permitieran pagar a 250 hombres a caballo armados, mandados por Bernat de Centelles, y otros 100 por Pedro Maça de Liçana, durante dos meses para combatir a los extranjeros que 
entraban en el reino a ayudar al conde de Urgel, ejércitos que luego estarán también en el asedio a Balaguer ${ }^{15}$.

Los diputados asumen también el sostenimiento de estas tropas, según acuerdan en los segundos capítulos que envían al rey, para ampliar la oferta que hace el reino de Valencia.

Las cantidades abonadas para liquidar el mes de prórroga del ejército ascienden, según el memorial que presenta Pedro Gil, a 8127 florines, 10 sueldos. A ellos hay que añadir 575 florines, 6 sueldos que se gastan en el viaje, custodia del dinero y salarios a diversos funcionarios. En esta última partida, Pedro Gil incluye la cantidad de 166 florines de quebranto de moneda, alegando que se le entregó la cantidad total de los 10.000 florines pesados de 1.000 en 1.000 y él tuvo que pagar pesando florín a florín y cuanto más de 100 en 100 florines, añadiendo que además le apremiaban las prisas de los que tenían que cobrar, encontrándose al fin con un déficit de 166 florines que ha de poner como gasto, ya que ha cuidado el dinero con más interés que si fuera suyo ${ }^{16}$.

Las cuentas que presentó al Parlamento fueron sometidas a las formalidades vigentes para las cuentas del General del Reino, según se acordó en los capítulos, y después de tomarle juramento sobre la veracidad del déficit, se dieron por buenas y se aprobaron.

Las actas del Parlamento terminan con la copia de la carta, de fecha 28 de diciembre de 1413, que los diputados envian al rey con mossen Ferrer, que le explicará largamente por qué no ha habido posibilidad de prorrogar un segundo mes el salario a los 500 hombres que estuvieron en el sitio de Balaguer.

Durante cuatro meses, el General del reino de Valencia ha sostenido un ejército que primero será de 300 hombres pero que posteriormente llegará a los 500, pudiendo encontrar reflejados en los libros de Clavería y Protocolos de esta institución corres-

15 ARV, Maestre Racional, libro 9813. Hay un cuadernillo, (8), en el que se contabiliza la administración de la oferta de 10.000 florines ofrecidos por el Brazo Real para sostenimiento de un ejército solicitado por Fernando I.

16 ARV, Real, libro 619 bis, fol. 38 y ss. 
pondientes a los años 1413 y 1414 , las cantidades que se manejan por estas circunstancias: sostenimiento de las tropas ${ }^{17}$, venta de las generalitats ${ }^{18}$ o de censales para recabar dinero, pago de intereses o de redención de censales ${ }^{19}$, salarios a los asesores y funcionarios que participan en las sesiones del Parlamento ${ }^{20}$, las cantidades que se abonan a los correos que llevan y traen cartas de Balaguer ${ }^{21}$, incluso con indemnizaciones por las buenas noticias que traen ${ }^{22}$. Así pues, el Parlamento aprobó un gasto de 18.000 florines en los primeros capítulos y en los segundos hasta 20.000 , si hubiera durado dos meses más el sitio de Balaguer, de los que no tuvo que invertir más que 10.000 florines, pero si se contabilizaran todas las cantidades invertidas por todos los conceptos, desde luego se llegó a cubrìr la oferta que se hizo al rey.

El reino de Valencia fue consecuente con su voto dado en Caspe y apoyó a Fernando I con generosidad y de forma voluntaria, cuando Jaime de Urgel, bien porque creyera que su derecho era prioritario al de Fernando de Antequera, bien porque los malos consejos de sus allegados lo alentaran, se rebeló contra la decisión de Caspe y presentó batalla al rey aragonés, muriendo, víctima de su rebeldía, en el castillo de Játiva.

17 ARV, Generalidad, libro 681 , fols. $87 v^{\circ}, 97 v^{\circ}, 98,101 v^{\circ}$ y ss. En esta nota y en las siguientes se da referencia de algunos de los muchos documentos en los que se contabilizan ingresos y pagos por causa de las dos ofertas hechas al Rey.

Ix ARV, Generalidad, libro 682, fol. 13.

19 ARV, Generalidad, libro 681, fol. $94 v^{\circ}$; Generalidad, libro 682, fol. 44 $v^{\circ}$; Generalidad, libro 2642, fol. $152 v^{\circ}$ y Generalidad, libro 4930 bis, fol. 724.

20 ARV, Generalidad, libro 681, fol. 97 y Generalidad, libro 2642, fol. $157 \mathrm{v}^{\circ}$.

21 ARV, Generalidad, libro 681, fol. 86 y ss, y Generalidad, libro 2642, fol. 131 y ss.

22 ARV, Generalidad, libro 681, fol. $95 v^{\circ}$. 


\section{APÉNDICE DOCUMENTAL}

1413, junio, 17, Valencia.

Los diputados del General de Valencia solicitan noticias a los jurados de la ciudad de Zaragoza sobre la invasión de tropas extranjeras en los territorios del reino de Aragón.

Als molts honorables e molt savis senyors los jurats de la ciutat de Saragoça.

Senyors molt honorables. Vista havem una carta vostra tramesa als jurats d'aquesta ciutat per la qual asats largament son asabentats d'algunes novitats seguides en aqueix Regne e de les gents d'armes stranyes que's speraben entrar per dapnejar les terres subiectes nostre ver Rey, princep e senyor, per la qual raho aquest Regne enten a fer algunes provisions en servey del dit senyor, be en defencio de la cosa publica, les quals vendrien a carrech del General del dit nostre Regne. E per provehir degudament en les dites coses sia a nosaltros necessari saber quant es lo nombre cert de les gents o companyes que's diu son enviades o deven entrar en aqueix regne de Arago, e on son a present, e les provisions que aqueix Regne hi a feyt, o enten certament fer, axi de asoldadar gents d'armes com altres, e si algunes altres novitats son aqui e en aqueix Regne seguides. Per tal, senyors mol honorables, vos pregam tan cordialment com podem, que de tot vos placia a nosaltres largament certifficar per manera que ab fonament de veritat en los dits affers puxam provehir segons lur exhigencia requira, e que per correu cuytat vos placia rescriure de tot largament, e haurem ho a notable plaher. E si per vos honorables senyors podem res açi complir scrivits nos francosament.

Scrit en Valencia a XVII de juny de mil CCCC XIII.

Los diputats del General del regne de Valencia a vostra honor aparellats.

ARV, Generalidad, libro 4930 bis, fol. 705.

1413, junio, 21, Valencia.

Capítulos que los diputados presentan al rey ofreciendo su ayuda para la defensa de sus territorios.

Primerament, los dits III staments Ecclesiastich, Militar e Reyal, no per ço que hi sien tenguts, mas de lur bon grat e francha liberalitat e ab sos expresses condicions, protestacions e salvaments dessus dits, e no sens aquells, ne per altra manera, les quals aci volem hever per expresses e en cascuns dels dits capitols deius scrits acorden, volen e 
ordenen que sien feyts e trameses en servey del dit senyor Rey per deffensio de sos regnes e terres CCC homens de cavall armats, pagats a III meses, los quals comencen a correr del jorn avant que faran la mostra.

Item, que a cascu dels dits homens d'armes sia donat sou ans de lur partida per tots los dits tres meses, a raho de VII solidos per cascun jorn, e que aquells sien tenguts fer lo servi que necessari sera a coneguda del senyor Rey, o de son primogenit, o del capita, e que no'ls sia donat acorriment ni extimes.

Item, que algun soldat no gos ne presumescha partirse del dit servi per alguna nececitat o causa sens licencia de son capita, sots pena de perdre lo roci e les armes e tant com es lo sou de hun mes, la qual pena sia applicada al General del dit Regne. E ultra aço, aquell aytal restituesqua al dit General, en tot cas, tot ço que reebut aura per sou del temps no servit.

Item, que lo dit sou sia donat e pagat a homens de cavall naturals o heretats o domiciliats dins lo regne de Valencia, o persones que ab aquells e en son servir stiguen, sens frau, e que a algun strany del dit Regne, si donchs no eren companyons dels dessus dits domiciliats o heretats en lo Regne, no sia donat del dit sou mentre que del dit Regne ne sien atrobats suficients a compliment dels dits CCC de cavall, e que aço 's faça a coneguda de aquells que faran lo dit pagament.

Item, que ans de compliment de paga fahedora als dits CCC de cavall sien tenguts fer e donar mostra de sos rocins e arnesos en poder de aquells qui faran los dits pagaments.

Item, que 'ls dits soldadats sien tenguts donar mostra de lurs armes e rocins, tota vegada e en qualsevol loch o lochs que al capita sera ben vist. E si 'I scriva de racio de casa del senyor Rey, o lochtinent o substituhit seu, o qualque altre volra entrevenir que ho puxa fer, empero sens tot dret de sou de hun dia o de salari, o de altra cosa que no sia dat al dit scriva de racio, lochtinent o substituhit, o altre per son offici, ni per son treball, ni en altra manera.

Item, que dels $\mathrm{CCC}$ homens de cavall sia capita lo molt egregi e spectable don Alfonso, duch de Gandia, al qual lo senyor Rey atorch plenera jurisdiccio, mer e mixt imperi, bastantment segons en semblants fets es acostumat de donar e fer.

Item, que co que costara lo sou dels dits $\mathrm{CCC}$ de cavall e les messions que per la dita raho se hauran a fer se pague per generalitats del dit regne de Valencia, en semblant forma e manera levador exhigidor que huy se leve e exhigeix lo general imposat, e que huy se leve e cull de present.

Item, que per ço com es pervengut que los dits sou e messions se paguen tot per generalitat de dit Regne, que per aço no sia feyt negun preiuhi en lo sdevenidor a algu dels dits Staments, ne a alguna universitat o singulars del dit Regne ne puxa esser tret a us ne a consequencia. 
Item, durant la dita subvencio algu que en aquella sia o hira, vullas que sia hom de paratge, ciutada o altre, no sia ne puxa esser request per lo senyor Rey de algun servi per empresa sua, o per altra raho o manera. E axi mateix que alguns Ordens de Cavalleria no puxen esser requests de algun servi de frontera de moros, ne altre servi de alguns durant la present subvencio.

Item, per ço que moneda puxa esser hauda prestament per pagar lo dit sou e messions lo dit senyor Rey e los qui fermen los presents capitols, com lo senyor Rey los haura fermats, atorgats e actorizats e ara per lavors donem ple e bastant poder als deputats del General del dit Regne o a la maior part de aquells, ab que ni haia de cascun Stament que puixen vendre e alienar sobre los damunt dits generals de regne de Valencia e sobre qualsevol dels dits Staments, universitats e singulars del dit Regne tants censals ab carta de gracia que 'Is preus de aquells basten a dehuyt milia florins, que son necessaris a pagament del dit sou, ab tots aquells pactes, penes, juraments, promissions e cauteles que $a b$ los compradors dels dits censals se poran convenir.

Item, que los dits censals sien quitats e les pensions de aquells pagades de les peccunies que exiran de les dites generalitats, les quals generalitats ultra aço perque ja huy son imposades e obligades, sien exhigides e levades en lo dit Regne, segon que huy se exhegeixen, e 's leven tant e tant longament tro que los dits censals que 's carregaran per los dits XVIII ${ }^{\mathrm{m}}$ florins de les dites generalitats puixen esser quitats e pagades les pensions e messions, menyscaptes e perdues, cas que algunes se 'n haien a fer.

Item, que dels fets de la present subvencio e levament de les generalitats, per no fer multiplicacio de officials, los clavaris del dit General e los diputats sien tenguts donar compte e raho en poder dels jutges qui ja per actes de cort son assignats de hoir los comptes de les generalitats que huy se leven e en temps passat se son levades, e que los dits jutges comptadors haien poder de diffinir los dits comptes en la forma e manera que han poder de diffinir los comptes de les dites generalitats que huy se leven e en temps passat se son levades.

Item, que lo senyor Rey, ni son primogenit, ne son governador o altre quelasevol official, per gran poder que haia, no's puixen entrametre de les coses en la present subvencio contengudes, ni de les coses tocants aquella, en qualsevol manera, per via de simple querela, o de appellacio, o recors justs o injusts ni en altra qualsevol manera.

Item, que lo senyor Rey e son primogenit, e'ls governadors del regne de Valencia, tota vegada que requests ne seran per los dits clavaris, diputats o administradors, sien tenguts de donar consell, favor e ajuda als sobredits en les coses de la present subvencio e toquants a aquella en qualsevol manera, en tal forma que les coses contengudes en los presents capitols vinguen a degut effecte e compliment.

Item, que 'I senyor Rey e son primogenit sien tenguts jurar e prome- 
tre servar e tenir, e fer servar e tenir, los presents capitols e totes les coses en aquells contengudes e contra aquells no venir, ni permeta venir per alguna manera, causa o raho.

Item, que totes e sengles cartes, letres e provisions reyals exidores dels presents capitols, e aquells, e a qualsevol de aquells expedients o necessaris sien fetes o liurades als dits dels III Staments del dit Regne, e a cascun dells, franques de tot dret de sagell e de la scrivania del senyor Rey.

Item, que 'Is dits III Staments fermen ad invicem carta de indempnitat, ço es, que si per los censals que seran carregats sera requesta, instada o feta execucio alguna contra algu o alguns de les altres Stament o Staments, los que no seran convenguts d'aquell Stament o dels altres, sien tenguts per lur part guardar de dan e servar indempne aquell $o$ aquells qui seran demanats o executats e que de aço sien ordenades bastants cartes de indempnitat, dictadores a consell dels honorables micer Guillem Caera e micer Francesch Blanch.

E lests e publicats per mi, dit notari, los sobre dits capitols en presencia e audiencia de tots los dessus nomenats e dels testimonis deius contenguts, de continent los dels dits Staments requeriren per mi Jacme de Vallseguer, notari dessus dit, de totes les dites coses esser feta e liurada carta publica, en testimoni de veritat.

Presents testimonis foren a les dites coses lo honrat micer Francesch Blanch, licenciat en leys, en Francesch Ferri, notari, ciutadans de Valencia, en Pere Grife, vehi de Morella e n’Alfonso de Taraçona, porter del senyor Rey.

ARV, Real, 619 bis, fols. $3 v^{\circ}$ y ss.

1413, julio, 30, Valencia.

El duque de Gandía hace la presentación del ejército, como determinan los capítulos de la promesa hecha al Rey.

Preterea, en lo dia de ditmenge, en lo qual era comptat trenta del mes de juliol, en l'any dessus dit de la Nativitat de Nostre Senyor MCCCC XIII, vers ora de vespres, davant les portes del Reyal del molt alt senyor Rey, foren personalment constituits los honorables frare Berenguer Vich, subdelegat del molt reverent Mestre de Muntesa, mossen Gil Sanccez Monyoç, lo molt noble mossen Eximen Perez de Arenos e los molt honorables mossen Pero Pardo de la Casta, micer GuiIlem Caera e en Pere Gil, substituit del honrat en Ferrer Ram, diputats qui dessus, e present e appellat en Bernat de Vallseguer, notari, en loch e per lo scriva de la dita Diputacio, en presencia de innumerable multitut de poble ajustat en la rambla denant lo dit Reyal, sobrevench lo molt egregi e expectable don Alfonso, duch de Gandía, e capita, qui 
dessus armat e vestint un bandell de carmesi brocat d'aur ab IIII trompetes a son senyal e tres ministres e hun standart ab son senyal detras, e ab dos pages. Apres lo qual, vench en son orde la gent deius nomenada, armats be e encavalcats ab sos rocins, los quals tots aplegats en la dita rambla, per mils esser vists cascu ab son armes e cavall, se meteren en regla fahent un rench per via de carrer, e regoneguts aquells per los dits diputats con fossen abtament e pertinent armats e lo dit senyor Duch agues pres de cascu lo sagrament e homenatge acostumat. E foren los qui donaren aqui mostra dels arneses e rocins per ordre los infraseguents... (Sigue la relación de todos los caballeros con sus acompañantes en número de 271).

ARV, Real, 619 bis, fol. 16 r. y v ${ }^{\circ}$. 